\title{
Coroidoretinopatia central serosa
}

\author{
Central serouschorioretinopathy
}

Pedro Duraes Serracarbassa

Doutor em Medicina pela Faculdade de Medicina da Universidade de São Paulo. Médico colaborador do Departamento de Oftalmologia, setor de retina e vítreo, do Hospital das Clínicas - Faculdade de Medicina da Universidade de São Paulo.

Endereço para correspondência: Av. Brig. Faria Lima, 1903 cj. 43 - São Paulo (SP) CEP 01452-001.

E-mail: serracar@brfree.com.br

\section{RESUMO}

O autor descreve os achados clínicos e novos métodos diagnósticos da coroidoretinopatia central serosa. Discute os estudos experimentais e clínicos relacionados à patogênese da doença e apresenta as alternativas de tratamento e prognóstico da coroidoretinopatia central serosa, baseado em levantamento bibliográfico.

Descritores: Doenças da coróide/diagnóstico; Doenças da coróide/patologia; Descolamento retiniano/diagnóstico; Angiofluoresceinografia; Diagnóstico diferencial; Epitélio pigmentado ocular; Prognóstico

Os aspectos clínicos da coroidoretinopatia central serosa (CCS) foram inicialmente descritos por Von Graefe* em $1886^{(1)}$. Cerca de cem anos depois, Maumenee ${ }^{(2)}$, utilizando-se da angiofluoresceinografia, observou que o descolamento seroso da retina na região macular era conseqüência do escape de contraste no epitélio pigmentado da retina (EPR).

\section{INCIDÊNCIA}

A CCS atinge preferencialmente indivíduos do sexo masculino (em $85 \%$ dos casos), na faixa etária de 20 a 45 anos. É rara em pacientes da raça negra, e pode apresentar formas atípicas quando atinge indivíduos de origem latina e oriental.

Pacientes com personalidade descrita como "perfil tipo A", caracterizados por altos níveis de tensão emocional e ansiedade, apresentariam predisposição para o desenvolvimento da CCS.

A doença é tipicamente unilateral, podendo acometer o olho adelfo em $10 \%$ dos casos de forma simultânea ou intercalada. Apesar da unilateralidade, são observadas alterações atróficas do EPR nos olhos contra-laterais em cerca de $1 / 3$ dos pacientes.

\section{PATOGÊNESE}

A patogênese da CCS permanece obscura. Trabalhos experimentais com eletroculografia demonstraram alterações difusas no EPR, representadas por mudanças no fluxo iônico através destas células ${ }^{(3-4)}$. Outros autores foram capazes de simular quadros de CCS em animais, através da injeção endovenosa de epinefrina e intravítrea de indometacina ${ }^{(5-6)}$.

Sibayan et al. (2000) relataram a apoptose de células do EPR através da exposição a altos níveis de epinefrina "in vitro"(7).

Os corticosteróides parecem estar envolvidos na gênese da CCS, uma

* Van Graefe (1886) apud ${ }^{(1)}$ 
vez que pacientes submetidos a tratamento com corticoterapia prolongada, gestantes e portadores da Síndrome de Cushing, apresentam maior predisposição para o desenvolvimento da doença ${ }^{(8-9)}$. Arndt et al. (2001) demostraram "in vitro" e "in vivo" os efeitos dos corticosteróides sobre as células do EPR através de estudo eletrofisiológico ${ }^{(10)}$.

$\mathrm{O}$ advento da indocianinografia verde trouxe novas perspectivas no conhecimento da CCS. Múltiplos pontos de vazamento na coriocapilar são observados no decorrer do exame, diferentemente dos raros pontos de escape vistos na angiofluoresceinografia ${ }^{(11)}$.

Esta observação sugere que o evento inicial da doença seja um aumento da permeabilidade da coriocapilar e posteriormente, uma descompensação do EPR.

\section{HISTOPATOLOGIA}

Os relatos histopatológicos da CCS são escassos. Gass, em 1977, observou a integridade da coriocapilar e EPR em um paciente portador da doença que teve seu olho enucleado após infarto agudo do miocárdio ${ }^{(1)}$. De Venecia, em 1982, relatou a presença de material protéico sub-retiniano em um paciente portador de múltiplos descolamentos da retina neurosensorial que veio a óbito após transplante renal ${ }^{(12)}$.

$\mathrm{O}$ autor sugeriu que a proteína seria resultante da exsudação da coriocapilar.

\section{QUADRO CLÍNICO}

Sintomas - A diminuição da acuidade visual é o principal sintoma da CCS. Esta pode variar de 0,8 a 0,1 e geralmente é corrigida com a adição de lentes positivas. Outros sintomas comumente relatados são: metamorfopsia, escotoma positivo, micropsia e alteração da visão de cores. Alguns casos podem apresentar-se assintomáticos devido à ocorrência de descolamentos serosos da retina extra-foveais.

Sinais - O sinal mais característico da CCS é a elevação da retina neuro-sensorial na região da fóvea. Este descolamento apresenta-se geralmente plano, bem delimitado e de pequenas dimensões (Figura 1), fato este que dificulta a observação em alguns casos. A melhor forma de diagnosticá-lo é através do exame cuidadoso da mácula pela biomicroscopia com lâmpada de fenda. A elevação dos vasos retinianos e o desvio da fenda de luz em forma de "arco" na superfície do descolamento auxiliam o diagnóstico. Pode-se observar, em alguns olhos, elevações pequenas, bem delimitadas, amareladas, situadas abaixo do descolamento da retina, que correspondem a descolamentos do EPR.

O líquido que ocupa o espaço sub-retiniano é geralmente translúcido, podendo ser turvo (fibrinoso) em $10 \%$ dos $\operatorname{casos}^{(13)}$.

Nas fases crônicas da doença, observam-se alterações do EPR abaixo ou próximas às áreas de retina elevada, caracteriza-

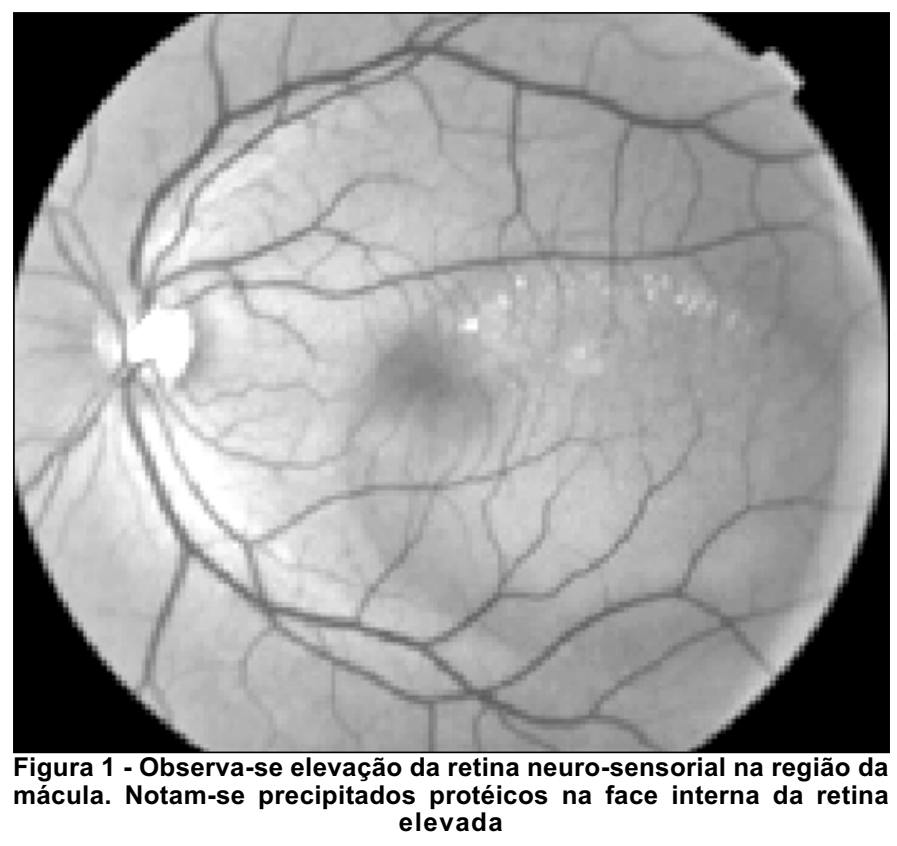

das por atrofia e mobilização de pigmento. Pode-se ainda encontrar, pequenos pontos amarelados na superfície interna da retina descolada, provavelmente relacionados a precipitados protéicos.

Outros achados menos freqüentes são: neovascularização de coróide ${ }^{(14)}$, edema macular cistóide ${ }^{(15)}$, rasgos do EPR ${ }^{(16)} \mathrm{e}$ hemorragia sub-retiniana ${ }^{(17)}$.

\section{FORMAS ATÍPICAS}

CCS crônica - atinge preferencialmente latinos e orientais e caracteriza-se pela presença de múltiplos pontos de vazamento originando áreas de descolamentos serosos recorrentes da retina. Inicialmente ocorrem em áreas extramaculares, criando extensas faixas de atrofia e mobilização de pigmento do EPR nos quadrantes inferiores. Observam-se, em alguns casos, descolamentos serosos de aspecto crônico nas regiões inferiores da retina. Posteriormente, desenvolvem-se descolamentos maculares recorrentes com possibilidade de diminuição significativa permanente da acuidade visual devido à atrofia do EPR na região da fóvea, edema cistóide de mácula e exsudação lipídica.

Descolamento de retina agudo bolhoso - Ocorre pela fusão de múltiplos descolamentos exsudativos da retina. Atinge principalmente indivíduos submetidos a corticoterapia prolongada sistêmica. Observam-se ainda múltiplos descolamentos do EPR e líquido sub-retiniano turvo em alguns pacientes. O quadro pode ser uni ou bilateral e apresenta prognóstico visual reservado devido às seqüelas atróficas do EPR.

CCS em mulheres - a doença é rara no sexo feminino (proporção 1:10 em relação ao sexo masculino) e comporta-se de 
forma semelhante àquela que acomete o sexo oposto. As mulheres portadoras de CCS estão geralmente acima dos 40 anos. A gestação e o uso de corticoterapia são fatores associados à doença e podem gerar quadros atípicos como descolamentos bolhosos bilaterais e exsudação de fibrina sub-retiniana.

CCS em idosos - pode-se observar a doença nos indivíduos nas últimas décadas de vida. O quadro clínico assemelha-se à forma clássica da doença, porém parece haver uma maior predisposição para o desenvolvimento da forma crônica. Nestes pacientes, o diagnóstico diferencial com a forma exsudativa da degeneração macular relacionada à idade é mandatório.

CCS e outras associações - encontram-se relatos associando a doença a dobras coriorretinianas ${ }^{(15)}$, retinose pigmentar(1), episclerite ${ }^{(1)}$ e associações sistêmicas como: hipertensão ${ }^{(27)}$, lupus eritematoso sistêmico ${ }^{(28)}$, doença de $\mathrm{Crohn}^{(29)}$, artrite reumatóide ${ }^{(30)}$, transplante renal ${ }^{(31)}$, transplante de medula óssea ${ }^{(32)}$, anemia hemolítica ${ }^{(15)}$, crioglobulinemia ${ }^{(15)}$ e síndrome antifosfolipídica ${ }^{(33)}$.

\section{DIAGNÓSTICO DIFERENCIAL}

O diagnóstico diferencial da CCS envolve desprendimentos maculares associados ou não a pontos de escape subretiniano.

As doenças que apresentam elevação da retina neurosensorial com vazamento incluem: Síndrome de Vogt-Koyanagi-Harada, hipertensão maligna, toxemia gravídica, neovascularização de coróide, Síndrome de efusão uveal idiopática, coroidopatia vascular polipoidal e tumores de coróide.

Os descolamentos serosos da retina sem pontos de escape englobam: descolamento de retina regmatogênico com roturas periféricas, buraco macular, fosseta do nervo óptico e tumores coroidais periféricos.

\section{ANGIOFLUORESCEINOGRAFIA}

A CCS apresenta diversos padrões no exame de angiofluoresceinografia (AFG). Nas fases precoces, observa-se ponto de vazamento de contraste no EPR, abaixo da retina elevada, que aumenta progressivamente de tamanho no decorrer do exame, promovendo um enchimento tardio do espaço subretiniano pela fluoresceína. Este padrão clássico pode manifestar-se de três formas distintas. O mais encontrado, em $60 \%$ dos casos, é o padrão em "mancha de tinta", no qual o ponto de escape aumenta gradativamente de maneira uniforme, corando as bordas de descolamento da retina nas fases finais do exame (Figura 2).

O padrão em "fumaça de chaminé" ocorre em $20 \%$ dos pacientes e é caracterizado pelo vazamento inicial do corante no EPR que posteriormente ascende para a região superior da retina elevada nas fases tardias (Figura 3 ). Acredita-se que o típico movimento do contraste para cima ocorra devido às correntes de convecção do líquido sub-retiniano ou pelo alto

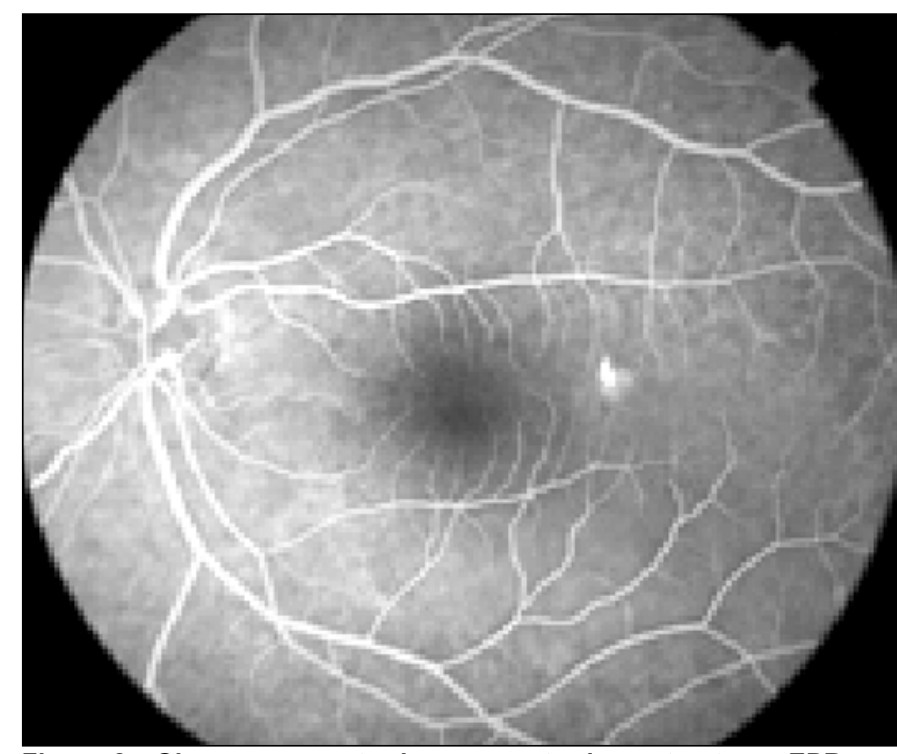

Figura 2 - Observa-se ponto de vazamento de contraste no EPR nas fases iniciais do exame de angiofluoresceinografia

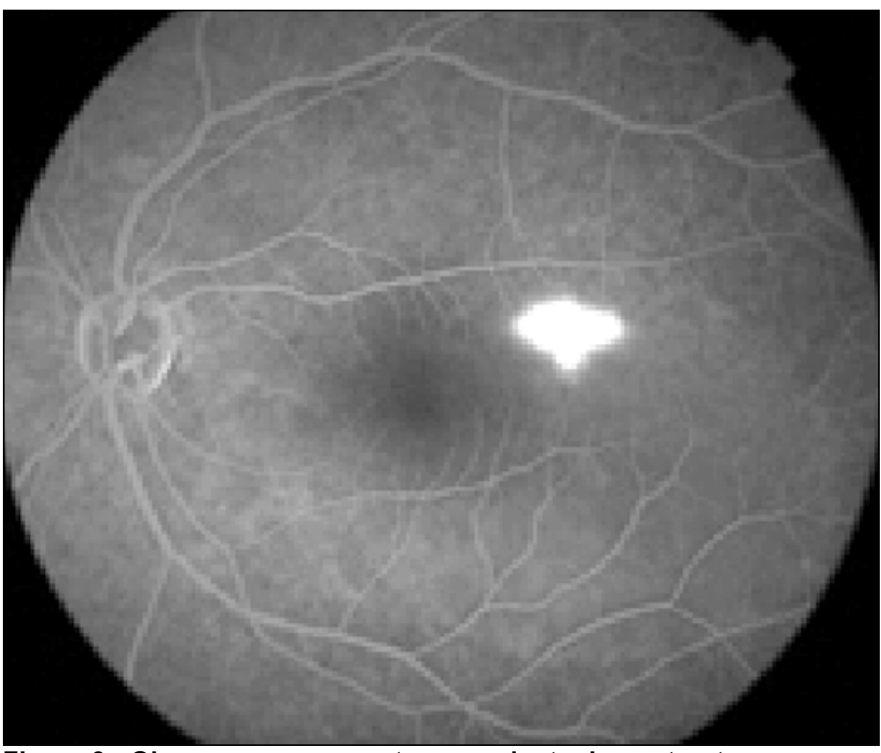

Figura 3 - Observa-se vazamento ascendente do contraste no espaço sub-retiniano nas fases tardias do exame de angiofluoresceinografia

peso molecular das proteínas que ocupam o espaço abaixo da retina em relação à fluoresceína.

Outro padrão angiofluoresceinográfico encontrado é o descolamento do EPR. Este se caracteriza pelo enchimento precoce, bem delimitado, do espaço sub-EPR seguido de ponto de escape do contraste.

Nos casos de CCS recorrente, são observados pontos de hiperfluorescência transmitida foveais ou justa-foveais correspondentes a áreas de atrofia do EPR.

Os padrões de vazamento não parecem ter valor prognóstico, porém acredita-se que o vazamento em "fumaça de chaminé" esteja relacionado à manifestação aguda da doença e a imagem em "mancha de tinta" associada às formas crônicas. 


\section{INDOCIANINOGRAFIA VERDE}

O exame de indocianinografia verde (ICG) na CCS apresenta características distintas da AFG. Na ICG pode-se observar o atraso no enchimento arterial seguido pela congestão capilar e venosa em determinadas áreas ${ }^{(18)}$.

Os principais achados são os múltiplos pontos de vazamento na coriocapilar encontrados nas fases média e tardia do exame. Estes pontos ocorrem nas áreas de congestão vascular e diferem do exame de AFG, no qual o vazamento geralmente é único ${ }^{(19-21)}$.

Outros sinais descritos são os múltiplos descolamentos do EPR “ocultos" representados por lesões hipofluorescentes $\operatorname{tardias}^{(22)}$. Observa-se ainda, a diminuição da fluorescência de fundo no pólo posterior nas fases tardias do exame ${ }^{(11)}$.

\section{TOMOGRAFIA ÓPTICA DE COERÊNCIA}

O exame de tomografia óptica de coerência (OCT) na CCS permite a observação da retina neuro-sensorial elevada na região da fóvea e, em alguns casos, do desprendimento do EPR. O espaço sub-retiniano apresenta baixa refletividade, podendo estar ocupado por massas de alta refletividade correspondentes a exsudatos fibrinosos ${ }^{(23)}$.

Autores descrevem ainda o aumento da espessura da retina descolada nas fases agudas da doença ${ }^{(24)}$.

\section{ELETRORRETINOGRAFIA}

São descritas alterações eletrofisiológicas nas fases agudas e crônicas da CCS. Observa-se diminuição significativa da amplitude da onda $a$ e $b$ e aumento da latência da onda $b$ nas fases agudas da doença. Estas alterações são comumente encontradas nos olhos contra-laterais e persistem com valores subnormais, mesmo após a resolução do descolamento da retina neuro-sensorial ${ }^{(25-26)}$.

\section{HISTÓRIA NATURAL}

A CCS apresenta, em geral, evolução benigna. Em 95\% dos casos, a acuidade visual corrigida atinge níveis melhores que 0,8 após 4 a 6 meses de aparecimento dos sintomas. Porém a grande maioria dos pacientes persiste com queixas visuais mínimas como micropsia, escotomas relativos, metamorfopsia, alteração da visão de cores e diminuição da sensibilidade de contraste. Nos $5 \%$ restantes, a visão permanece pior que 0,8 após seis meses de observação. As recidivas são freqüentes, acometendo cerca de 20 a $30 \%$ dos olhos.

Acredita-se que o tempo de duração do descolamento da retina, a proximidade do ponto de vazamento da fóvea e as alterações atróficas do EPR sejam fatores de mau prognóstico visual.

\section{TRATAMENTO}

A falta de conhecimento da gênese da CCS dificulta o tratamento do fator causal da doença. Autores sugerem prováveis respostas satisfatórias com o uso de beta-bloqueadores orais ${ }^{(14)}$, porém a falta de trabalhos com grupos-controle dificulta a análise dos reais efeitos desta medicação. A associação de altos níveis de corticosteróides sanguíneos com quadros de CCS descritos na literatura contra-indica o uso destas substâncias no tratamento da doença.

O objetivo da fotocoagulação no tratamento da CCS é diminuir o tempo de duração da doença ${ }^{(34)}$. Não existe comprovação de que o laser contribua para a recuperação visual final destes pacientes ${ }^{(35)}$. Desta forma, opta-se pelo tratamento nas seguintes situações: persistência do descolamento da retina após 4 meses, recidivas sem melhora após 1 mês, formas atípicas e necessidade profissional do paciente.

A técnica de aplicação do laser envolve o reconhecimento do ponto de vazamento no exame angiofluoresceinográfico. Focaliza-se então este ponto de escape e aplica-se o laser com baixa intensidade, mira pequena $(50 \mu)$ e mínima duração $(0,1 \mathrm{~s})$. O objetivo é repopular a região de vazamento com células do EPR sadias. Evita-se o tratamento de pontos de escape situados dentro de 1/4 de diâmetro papilar da fóvea.

A diminuição do descolamento ocorre geralmente de 1 a 3 semanas após a fotocoagulação. Nos casos resistentes ao tratamento, sugere-se a investigação diagnóstica de prováveis pontos de escape situados fora do pólo posterior e doenças que podem simular quadros de CCS conforme citadas no diagnóstico diferencial, principalmente membranas neovasculares de coróide e coroidopatia vascular polipoidal ${ }^{(36)}$.

Os riscos da fotocoagulação na região justa-foveal são consideráveis. O principal é o desenvolvimento de neovascularização de coróide que ocorre em $5 \%$ dos casos tratados. Questiona-se se esta complicação seria um efeito danoso direto do laser sobre o complexo EPR-membrana de Brüch-coriocapilar ou um estímulo térmico sobre uma membrana neovascular coroidal pré-existente.

Outras possíveis complicações da fotocoagulação são: membrana pré-retiniana, buraco macular, queimadura acidental da fóvea, hemorragia e depósitos protéicos na fóvea ${ }^{(37)}$.

\section{ABSTRACT}

The author describes the clinical features and new diagnostic methods of central serous chorioretinopathy. He discusses experimental and clinical studies related to the pathogenesis of the disease and presents therapy choices and prognosis of central serous chorioretinopathy, based on reference survey.

Keywords: Choroid diseases/diagnosis; Choroid diseases/ pathology; Retinal detachment/diagnosis; Fluorescein angiography; Differential diagnosis; Pigment epithelium of eye; Prognosis 


\section{REFERÊNCIAS}

1. Gass JDM. Stereoscopic atlas of macular diseases: diagnosis and treatment. 3a ed. St. Louis: Mosby; 1987. p.46-59.

2. Maumenee AE. Symposium: macular diseaes. Pathogenesis. Trans Am Acad Ophthalmol Otolaryngol 1965;69:691-9.

3. Marmor MF. New hypothesis on the pathogenesis and treatment of serous retinal detachment. Graefes Arch Clin Exp Ophthalmol 1988;226:548-52.

4. Spitznas M. Pathogenesis of central serous retinopathy: a new working hypothesis. Graefes Arch Clin Exp Ophthalmol 1986;224:321-4.

5. Yoshioka H, Katsume Y, Akune H. Experimental central serous chorioretinopathy in monkey eyes: fluorescein angiographuc findings. Ophthalmologica 1982;185:168-178.

6. Watanabe S, Ohtsuki K. Experimental central serous chorioretinopathy. Acta Soc Ophthalmol Jpn 1979;83: 808-17.

7. Sibayan S A, Kobuch K, Spiegel D, Eckert E, Leser R, Monzer J, et al. Epinefrine, but not dexamethasone, induces apoptosis in retinal pigment epithelium cells in vitro: possible implications on the pathogenesis of central serous chorioretinopathy. Graefes Arch Clin Exp Opthalmol 2000;238:515-9.

8. Wakakura M, Ishikawa S. Evaluation of corticosteriod treatment of central serous chorioretinopathy. Jpn J Clin Ophthalmol 1980;34:123-9.

9. Tittl MK, Spaide RF, Wong D, Pilotto E, Yannuzzi LA, Fisher YL, Guyer $\mathrm{DR}$, et al. Systemic findings associated with central serous chorioretinopathy. Am J Ophthalmol 1999;128:63-8.

10. Arndt C, Sari A, Ferre M, Parrat E, Courtas D, De Seze J, Hache J, et al. Electrophysiological effects of corticosteroids on the retinal pigment epithelium. Invest Ophthalmol Vis Sci 2001;42:472-5.

11. Guyer DR, Yannuzzi LA, Slakter JS, Sorenson JA, Ho A, Orlock D. Digital indocyanine green videoangiography of central serous chorioretinopathy [commented on Arch Ophthalmol 1995;113:701-2]. Arch Ophthalmol 1994;112:1057-62.

12. De Venecia G. Fluorescein angiographic smoke stack. In: Verhoeff Zimmerman Society. Meeting. Washington, DC, April 24-25, 1982. Available from: URL: http://pathology.mc.duke.edu/EyePath/vs1982.htm\#top

13. Pimentel SLG, Abujamra S. Corioretinopatia central serosa com líquido turvo: relato de dezoito casos. Arq Bras Oftalmol 1995;58:407-11.

14. Gomolin JES. Choroidal neovascularization and central serous chorioretinopathy. Can J Opthalmol 1986;24:20-3.

15. Gass JDM. Stereoscopic atlas of macular diseases: diagnosis and treatment. $4 \mathrm{a}$ ed. St. Louis: Mosby; 1997. p.65-6.

16. Shanmugan MP, Bhende M. Retinal pigments epithelium tears associated with central serous chorioretinopathy. Indian J Ophthalmol 2000;48:315-7.

17. Kwok AK, Lai TY, Cheng AC, Lam DS. Massive subretinal haemorrhage associated with central serous chorioretinopathy. Eye 2001;15:121-3.

18. Piccolino FC, Borgia L. Central serous chorioretinopathy and indocyanine green angiography. Retina 1994;14:231-42.
19. Piccolino FC, Borgia L, Zinicola E, Zingirian M. Indocyanine green angiographic findings in central serous chorioretinopathy. Eye 1995;9(Pt 3):324-32.

20. Menchini U, Virgili G, Lanzetta P, Ferrari E. Indocianine green angiography in central serous chorioretinopathy. ICG angiography in CSC. Int Ophthalmol 1997;21:57-69.

21. Nishiyama Y, Mori K, Mutayama K, Yoneya S. Quantitative analysis of indocyanine green angiographic image in central serous chorioretinopathy. Jpn J Ophthalmol 2001;45:116-8.

22. Prunte C. Indocyanine green angiographic findings in central serous chorioretinopathy. Int Ophthalmol 1995;19:77-82.

23. Iida T, Hagimura N, Sato T, Kishi S. Evaluation of central serous chorioretinopathy with optical coherence tomography. Am J Ophthalmol 2000;129:16-20.

24. Bek T, Kandi M. Quantitative anomaloscopy and optical tomography scanning in central serous chorioretinopathy. Acta Ophthalmol Scand 2000;78:632-7.

25. Chappelow AV, Marmor MF. Multifocal electroretinogram abnormalities persist following resolution of central serous chorioretinopathy. Arch Ophthalmol 2000;118:1211-5.

26. Suzuki K, Hasegawa S, Usui T, Ichibe M, Takada R, Takagi M, et al. Multifocal electroretinogram in central serous chorioretinopathy. Jpn J Ophthalmol 2000;44:571-3.

27. Tittl MK, Spaide RF, Wong D, Pilotto E, Yannuzzi LA, Fisher YL, et al. Systemic findings associated with central serous chorioretinopathy. Am J Ophthalmol 1999;128:63-8.

28. Carpenter MT, O'Boyle JE, Enzenauer RN, Enzenauer RJ, Waterhourse WJ. Choroiditis in systemic lupus erythematous. Am J Ophthalmol 1994;117:535-6.

29. Schreiber JB, Lakhanpal V, Nasrallah SM. Crohn's disease complicated by idiopathic central serous chorioretinopathy with bullous retinal detachment. Dig Dis Sci 1989;34:118-22.

30. Williamson J, Nuki G. Macular lesions during systemic therapy with depot tetracosactrin. Br J Ophthalmol 1970;54:405-9.

31. Friberg TR, Eller AW. Serous retinal detachment resembling central serous chorioretinopathy following organ transplantation. Graefes Arch Clin Exp Ophthalmol 1990;228:305-9.

32. Fawzi AA, Cunningham Jr. ET. Central serous chorioretinopathy after bone marrow transplantation. Am J Ophthalmol 2001;131:804-5.

33. Costen MT, Olson J. Central serous chorioretinopathy may be a manifestation of the primary antiphospholipid syndrome. Br J Ophthalmol 2000;84:667-8.

34. Robertson DM. Argon laser photocoagulation treatment in central serous chorioretinopathy. Ophthalmology 1986;93:972-4.

35. Charollais RH, Alder H, Ferber A, Koniecki J, Sell C, Baserga R. Laser treatment of diffuse retinal pigment epitheliopathy. Eur J Ophthalmol 1992;2:103-14.

36. Yannuzzi LA, Freund KB, Golbaum M, Scassellati-Sforzolini B, Guyer DR, Spaide RF, et al. Polypoidal choroidal vasculopathy masquerading as central serous chorioretinopathy. Ophthalmology 2000;107:767-7.

37. Costa VP, Pimentel SLG, Warren SHC, Abujamra S. Fotocoagulação com laser de argônio no tratamento da retinopatia central serosa. Arq Bras Oftalmol 1991;54:213-6.

\title{
ABO ELETRÔNICO
}

\author{
Novo site
}

Acesso: http://www.abonet.com.br 